\title{
Long-term outcomes of prediction error after combined phacoemulsification and trabeculectomy in glaucoma patients
}

\author{
Yeon Soo Kang, Mi Sun Sung, Hwan Heo, Yong Sok Ji and Sang Woo Park ${ }^{*}$
}

\begin{abstract}
Background: To investigate long-term outcomes of prediction error after phacotrabeculectomy and to determine risk factors that may cause unstable prediction error after phacotrabeculectomy in glaucoma patients.

Methods: A total 120 eyes of 120 patients who had underwent uncomplicated phacotrabeculectomy (combined group) or phacoemulsification (phaco-only group) were included. Best-corrected visual acuity (BCVA), intraocular pressure (IOP) were measured before and after surgery, and anterior segment parameters including anterior chamber depth (ACD), lens vault (LV), and anterior vault (AV) measured using anterior-segment optical coherence tomography were compared between the two groups. The mean absolute error (MAE) at 3, 6, 12, and 24 months postoperatively were compared. Risk factors associated with unstable prediction error (MAE $\geq 0.5$ ) were investigated in the combined group.

Results: In both groups, BCVA was improved and IOP was decreased significantly. MAE at 3, 6, 12, 24 months postoperatively were not significantly different between two groups. The risk factors for unstable prediction error after 12 months of phacotrabeculectomy were old age and LV. Whereas, the only factor predicting unstable prediction error after 24 months of phacotrabeculectomy was LV. The cut-off value of LV for predicting unstable refractive error analyzed by the ROC curve was $0.855 \mathrm{~mm}$.

Conclusions: Phacotrabeculectomy may be an effective treatment with stable long-term outcomes of prediction error similar to phacoemulsification in patients with glaucoma. However, elderly patients or patients with large LV may be predisposed to unstable prediction error after phacotrabeculectomy.
\end{abstract}

Keywords: Phacotrabeculectomy, Prediction error, Anterior segment parameters, Medically uncontrolled glaucoma, Lens vault

\section{Background}

When planning the surgery of a patient with cataract and medically uncontrolled glaucoma, it becomes difficult to decide whether to perform trabeculectomy and phacoemulsification at the same time or to perform phacoemulsification after trabeculectomy. It has already been reported that axial length (AL) and

\footnotetext{
* Correspondence: exo70@naver.com

Department of Ophthalmology, Chonnam National University Medical School and Hospital, 42 Jebong-ro, Dong-gu, Gwangju 61469, South Korea
}

anterior chamber depth (ACD) decrease [1-3] and keratometry changes [4-6] after trabeculectomy. Because these parameters play an important role in determining the intraocular lens (IOL) power [7-10], there is a possibility that postoperative prediction errors may increase if the IOL power calculation formula used for phacoemulsification is used when performing phacotrabeculectomy [11, 12].

If phacoemulsification is performed with uncontrolled intraocular pressure (IOP), surgery becomes difficult due 
to high posterior pressure [13]. Complications such as hypotony, hyphema, shallow anterior chamber may occur after phacotrabeculectomy [14, 15], and the frequency of complications is higher than that of phacoemulsification alone [16, 17]. Nevertheless, there are several advantages of phacotrabeculectomy such as improving visual acuity after surgery, minimizing postoperative IOP spikes, and reducing morbidity that can occur in two stage operations [18-20]. Since the success rate has been increased by the using of mitomycin C [21, 22], phacotrabeculectomy is widely performed in recent clinical trials.

As the calculations for IOL power improve due to the development of preoperative evaluations and surgical techniques, refractive errors are becoming more predictable [22, 23]. There is increasing interest in reducing prediction errors after phacotrabeculectomy because trabeculectomy can affect refractive errors after phacoemulsification. Recently, several studies focusing on outcomes of prediction error after phacotrabeculectomy have been published [11, 18, 22-25].

However, most of the previous studies examined short-term outcomes of prediction error less than 6 months, and no studies have investigated risk factors that can cause unstable prediction error after phacotrabeculectomy. Therefore, the present study aims to compare the long-term outcomes of prediction error of phacotrabeculectomy and phacoemulsification, and to determine the factors that predict unstable refractive error after phacotrabeculectomy.

\section{Methods}

\section{Subjects}

We retrospectively reviewed medical records of patients who had underwent uncomplicated phacotrabeculectomy (combined group) or uncomplicated phacoemulsification (phaco-only group) between September 2015 and December 2018. Patients underwent phacotrabeculectomy when IOP was not controlled even with the maximal tolerated medical treatment. Patients who were able to follow-up for more than 1 year were included, and exclusion criteria included prior keratorefractive surgery, ocular disease that may affect refractive errors except glaucoma and cataract, IOL in the ciliary sulcus or sclera fixation of IOL, and bleb needling after phacotrabeculectomy. If both eyes of a patient satisfied these criteria, one eye was chosen randomly. Ethical approval was obtained from the Chonnam National University Hospital Institutional Review Board, and the study protocol followed the guidelines of the Declaration of Helsinki.

\section{Data collection}

All patients underwent preoperative evaluations including best-corrected visual acuity (BCVA), manifest refraction using autorefractor keratometer (ARK; Topcon KR-8900, Topcon, Tokyo, Japan), IOP using Goldmann applanation tonometry, and slit-lamp examination. For comparison, BCVA was converted to logarithm of the minimum angle of resolution (LogMAR) and the refraction value was converted to spherical equivalent (SE) by adding spherical power to $1 / 2$ of cylinder power. And 12 months after the surgery, BCVA and IOP were measured to investigate the effect of the two operations on visual acuity and IOP.

K-value using ARK and axial length (AL) using partial coherence interferometry (Lenstar ${ }^{\circ}$, Haag-Streit, Bern, Switzerland) were measured, and SRK-T formula was used to calculate IOL power and predicted refractive errors. Additionally, anterior segment parameters were measured by using anterior-segment optical coherence tomography (AS-OCT) device (Visante ${ }^{\circ}$, Carl Zeiss Meditec, Dublin, CA). A single examiner (S.W.P) selected the best images with no motion artifacts, good visibility of the scleral spur, and no image artifacts from the eyelids. And then, two independent examiners (Y.S.K and M.S.S) who were blinded to other clinical information analyzed images using custom software (Iridocorneal module, Carl Zeiss Meditec). Anterior chamber depth (ACD) was defined as the distance between the center of posterior corneal surface and anterior lens surface, lens vault (LV) was defined as the maximum perpendicular distance between the anterior lens surface and horizontal line connecting the two sclera spurs, and anterior vault (AV) was defined as the sum of ACD and LV [26].

\section{Surgical technique}

Surgical procedures were performed by a single surgeon (S.W.P) under topical or retrobulbar anesthesia. Standard phacoemulsification was used to remove the cataract through a temporal $2.8 \mathrm{~mm}$ clear corneal incision. In all cases, Acrysof SN60WF (Alcon, Fort Worth, TX) IOL was implanted in the capsular bag.

For a phacotrabeculectomy, a fornix-based conjunctival flap and rectangular shaped half-thickness scleral flap were created. Phacoemulsification and IOL implantation were performed through clear corneal incision at a different site with trabeculectomy. A sponge soaked in $0.04 \%$ mitomycin $\mathrm{C}$ was placed under the conjunctiva and Tenon's capsule on the sclera for $3-5$ min according to the discretion of the surgeon and based on the patient characteristics. Sclerotomy and peripheral iridectomy were performed. Finally, the scleral flap was sutured with 2 interrupted sutures using 10-0 nylon and the conjunctiva was closed.

\section{Outcome measures}

Mean absolute error (MAE) was defined as the absolute value of the difference between predicted refractive 
errors and postoperative refractive errors. MAE at 3, 6, 12 , and 24 months postoperatively were compared between two groups. The combined group was further divided into two subgroups based on the prediction errors: stable prediction error $(\mathrm{MAE}<0.5$ diopters $[\mathrm{D}])$ and unstable prediction error (MAE $\geq 0.5 \mathrm{D}$ ). Risk factors associated with unstable prediction error were investigated at 12 and 24 months postoperatively. The number of glaucoma medications used preoperatively and 12 and 24 months postoperatively were also compared to determine the IOP lowering effect of phacotrabeculectomy.

\section{Statistical analysis}

SPSS version 26.0 (SPSS Inc., Chicago, IL) was used for all statistical analyses. Independent $t$-test or MannWhitney $U$ test was used to compare the continuous data of the two groups, and paired $t$-test was used to compare the values before and after surgery in the same group. Chi-square test was used to compare categorical data, and logistic regression analysis was used to investigate risk factors that may cause unstable prediction error. Variables with a significance level at $P<0.1$ in the univariable analysis were included in the multivariable analysis. The receiver operating characteristic (ROC) curve analysis was used to determine the optimal cut-off value of risk factor predicting unstable refractive error. The ability of the cut-off value to predict accurately is represented by the area under the curve (AUC). $P$ values less than 0.05 were considered statistically significant.

\section{Results}

A total 120 eyes of 120 patients were enrolled in this study, and 60 eyes were included in each group. The subject's demographics and baseline characteristics of the included eyes are summarized in Table 1 . Since patients in the combined group had medically uncontrolled glaucoma, the mean preoperative IOP was $29.60 \pm 10.04$ $\mathrm{mmHg}$, which was significantly higher than those in phaco-only group $(P<0.001)$. ACD was significant smaller in the combined group than that of the phacoonly group $(P=0.018)$. There were no significant differences in age, sex, preoperative BCVA, AL, LV, and AV.

In both combined group and phaco-only group, BCVA was significantly improved $(P=0.001$ and $P<0.001$, respectively) and IOP was significantly decreased $(P<$ 0.001 and $P<0.001$, respectively) at 12 months postoperatively (Table 2). The phaco-only group tended to show better postoperative BCVA than the combined group, but there was no statistical significance $(P=0.065)$. Mean postoperative IOP of the combined group was $13.35 \pm 3.12 \mathrm{mmHg}$, which remained stable until 12 months after phacotrabeculectomy. MAE at 3, 6, 12, and 24 months postoperatively were not significantly different between the two groups $(P=0.072, P=0.117, P=$ 0.226 , and $P=0.083$, respectively) (Table 3 ). That is, outcomes of prediction error after phacotrabeculectomy were similar with that of the phacoemulsification alone until 24 months postoperatively.

Table 4 showed characteristics of the stable and unstable prediction error subgroups in the combined group at 12 and 24 months postoperatively. Patients in the unstable subgroup were significantly older $(P=0.024)$ and had a larger $\operatorname{LV}(P=0.038)$ and larger $\operatorname{AV}(P=0.041)$ than those in the stable subgroup at 12 months postoperatively. And patients in the unstable subgroup had significantly shallower ACD $(P=0.040)$ and larger LV $(P=$ 0.010 ) than those in the stable subgroup at 24 months postoperatively.

The univariable analysis showed that old age $(\mathrm{OR}=$ $1.060, P=0.031)$, large LV $(\mathrm{OR}=6.838, P=0.045)$, and large AV $(\mathrm{OR}=4.873, P=0.047)$ were associated with unstable prediction error at postoperative 12 months after combined surgery. The multivariable analysis showed that old age $(\mathrm{OR}=1.069, P=0.030)$ and large $\mathrm{LV}(\mathrm{OR}=5.687, P=0.029)$ were risk factors of unstable prediction error at 12 months postoperatively (Table 5 ).

Table 1 Subject's demographics and baseline characteristics of included eyes

\begin{tabular}{llll}
\hline Variables & Combined group $(\boldsymbol{n}=\mathbf{6 0})$ & Phaco-only group $(\boldsymbol{n}=\mathbf{6 0})$ & $\boldsymbol{P}^{\text {value }}$ \\
\hline Age (years) & $65.65 \pm 11.02$ & $67.83 \pm 8.88$ & $0.234^{\mathrm{a}}$ \\
Sex (male/female) & $34 / 26$ & $30 / 30$ & $0.464^{\mathrm{b}}$ \\
Baseline BCVA (LogMAR) & $0.67 \pm 0.82$ & $0.47 \pm 0.58$ & $0.124^{\mathrm{a}}$ \\
Baseline IOP (mmHg) & $29.60 \pm 10.04$ & $14.52 \pm 2.50$ & $<0.001^{\mathrm{a}}$ \\
AL (mm) & $23.16 \pm 1.04$ & $23.26 \pm 1.17$ & $0.337^{\mathrm{a}}$ \\
ACD (mm) & $2.25 \pm 0.48$ & $2.48 \pm 0.56$ & $0.018^{\mathrm{a}}$ \\
LV (mm) & $0.82 \pm 0.36$ & $0.71 \pm 0.38$ & $0.090^{\mathrm{a}}$ \\
AV (mm) & $3.06 \pm 0.40$ & $3.19 \pm 0.38$ & $0.089^{\mathrm{a}}$ \\
\hline BCVA
\end{tabular}

$B C V A$ best-corrected visual acuity, LogMAR logarithm of the minimum angle of resolution, $I O P$ intraocular pressure, $A L$ axial length, $A C D$ anterior chamber depth, $L V$ lens vault, $A V$ anterior vault

${ }^{a}$ Independent t-test for combined group and phaco-only group

${ }^{\mathrm{b}}$ Chi-square test for combined group and phaco-only group 
Table 2 Visual outcomes and IOP reduction at 12 months postoperatively

\begin{tabular}{|c|c|c|c|}
\hline Variables & Combined group $(n=60)$ & Phaco-only group $(n=60)$ & $P$ value $^{a}$ \\
\hline \multicolumn{4}{|l|}{ Baseline } \\
\hline BCVA (LogMAR) & $0.68 \pm 0.82$ & $0.47 \pm 0.58$ & 0.124 \\
\hline IOP $(\mathrm{mmHg})$ & $29.60 \pm 10.04$ & $14.52 \pm 2.50$ & $<0.001$ \\
\hline \multicolumn{4}{|l|}{ Postoperative } \\
\hline BCVA (LogMAR) & $0.48 \pm 0.86$ & $0.24 \pm 0.56$ & 0.065 \\
\hline IOP $(\mathrm{mmHg})$ & $13.35 \pm 3.12$ & $13.23 \pm 2.20$ & 0.814 \\
\hline \multicolumn{4}{|l|}{$P$ value $^{\mathrm{b}}$} \\
\hline BCVA & 0.001 & $<0.001$ & \\
\hline IOP & $<0.001$ & $<0.001$ & \\
\hline
\end{tabular}

$I O P$ indicates intraocular pressure, BCVA best-corrected visual acuity, LogMAR logarithm of the minimum angle of resolution

${ }^{a}$ Independent $t$-test for combined group and phaco-only group

${ }^{b}$ Paired $t$-test for baseline and postoperative values

At postoperative 24 months, univariable and multivariable analysis determined that only large LV $(\mathrm{OR}=$ 19.647, $P=0.024$ ) was associated with unstable prediction error after combined surgery (Table 6).

The scatter plot of LV and MAE at 12 and 24 months postoperatively showed their relationships (Fig. 1). ROC curve analysis was performed to determine the optimal cut-off value of the LV that can predict unstable refractive errors (Fig. 2). The AUC of LV at 12 months postoperatively was 0.619 and the AUC of LV at 24 months postoperatively was 0.689 . In both ROC curves, the cutoff value of LV was $0.855 \mathrm{~mm}$.

The patients in the combined group used an average of 2.8 medications preoperatively. The needs for glaucoma medication were significantly reduced to average of 1.12 medications at 12 months $(P<0.001)$ and average of 1.27 medications at 24 months $(P<0.001)$ postoperatively.

\section{Discussion}

Cataract surgery has the advantage of quick visual recovery and relatively low complications [27-29]. Since cataract surgery alone has an effect of reducing IOP in patients with angle closure glaucoma (ACG) $[16,17,30]$ and open angle glaucoma (OAG) [31-33], phacoemulsification plays an important role in the treatment of glaucoma. In patients with ACG, phacoemulsification significantly deepens the ACD and resolves angle crowding. Because these changes are small in patients with OAG, the exact mechanism why IOP decreases after cataract surgery in patients with OAG remains controversial [29]. Some studies reported that both changes of angle configuration and trabecular meshwork or extracellular matrix remodeling are involved [27, 28]. In the current study, the fact that IOP significantly decreased postoperatively in the phaco-only group also supports previous studies.

Preoperatively, the ACD of the combined group was significantly shallower than that of the phaco-only group. This finding can be explained by the prevalence of ACG (34 eyes) in the combined group, whereas the phaco-only group had twelve patients with ACG. Previous studies reported that the refractive errors of patients with ACG were difficult to predict [34, 35], but our result showed relatively stable prediction error despite the large number of patients with ACG in the combined group.

Recently, the indications of phacotrabeculectomy are as follows: i) medically uncontrolled glaucoma, ii) tolerance of glaucoma medications, iii) postoperative IOP spikes may worsen visual field damage, iv) suspected compliance of glaucoma medications [15-17, 36]. Several studies reported that the IOP lowering effect of phacotrabeculectomy was superior to that of phacoemulsification alone in patients with glaucoma [36-38]. In the present study, the mean preoperative

Table 3 Outcomes of prediction error of the combined group and phaco-only groups

\begin{tabular}{llll}
\hline Variables & Combined group $(\boldsymbol{n}=\mathbf{6 0})$ & Phaco-only group $(\boldsymbol{n}=\mathbf{6 0})$ & $\boldsymbol{P}$ value \\
\hline MAE at 3 months postoperatively (D) & $0.69 \pm 0.47$ & $0.54 \pm 0.47$ & 0.072 \\
MAE at 6 months postoperatively (D) & $0.66 \pm 0.60$ & $0.52 \pm 0.32$ & 0.117 \\
MAE at 12 months postoperatively (D) & $0.59 \pm 0.44$ & $0.49 \pm 0.43$ & 0.226 \\
MAE at 24 months postoperatively (D) ${ }^{\text {b }}$ & $0.63 \pm 0.44$ & $0.47 \pm 0.36$ & 0.083 \\
\hline
\end{tabular}

MAE mean absolute error, $D$ diopters

andependent $t$-test for combined group and phaco-only group

${ }^{b}$ Only forty-seven patients were included in each of groups at 24 months postoperatively 
Table 4 Characteristics of the stable and unstable subgroups in combined group at 12 and 24 months postoperatively

\begin{tabular}{|c|c|c|c|c|c|c|}
\hline \multirow[t]{2}{*}{ Variables } & \multicolumn{3}{|c|}{12 months postoperatively } & \multicolumn{3}{|c|}{24 months postoperatively } \\
\hline & $\begin{array}{l}\text { Stable subgroup } \\
(n=30)\end{array}$ & $\begin{array}{l}\text { Unstable subgroup } \\
(n=30)\end{array}$ & $P$ value & $\begin{array}{l}\text { Stable subgroup } \\
(n=20)\end{array}$ & $\begin{array}{l}\text { Unstable subgroup } \\
(n=27)\end{array}$ & $P$ value \\
\hline Age (years) & $62.47 \pm 10.68$ & $68.83 \pm 10.58$ & $0.024^{a}$ & $62.40 \pm 11.77$ & $66.40 \pm 10.55$ & $0.222^{c}$ \\
\hline Sex (male/female) & $17 / 13$ & $17 / 13$ & $1.000^{b}$ & $12 / 8$ & $16 / 11$ & $0.599^{b}$ \\
\hline Type of glaucoma (ACG/OAG) & $17 / 13$ & $17 / 13$ & $1.000^{b}$ & $9 / 11$ & $17 / 10$ & $0.177^{b}$ \\
\hline Baseline BCVA (LogMAR) & $0.61 \pm 0.82$ & $0.74 \pm 0.82$ & $0.530^{a}$ & $0.87 \pm 1.05$ & $0.44 \pm 0.39$ & $0.197^{c}$ \\
\hline Postoperative BCVA (LogMAR) & $0.40 \pm 0.76$ & $0.57 \pm 0.95$ & $0.449^{a}$ & $0.59 \pm 10.7$ & $0.36 \pm 0.60$ & $0.334^{c}$ \\
\hline Baseline IOP (mmHg) & $31.87 \pm 10.50$ & $27.33 \pm 9.18$ & $0.080^{a}$ & $30.15 \pm 9.23$ & $29.37 \pm 10.01$ & $0.384^{c}$ \\
\hline Postoperative IOP (mmHg) & $13.50 \pm 2.67$ & $13.20 \pm 3.57$ & $0.714^{a}$ & $13.75 \pm 2.86$ & $13.48 \pm 3.41$ & $0.312^{c}$ \\
\hline $\mathrm{AL}(\mathrm{mm})$ & $23.11 \pm 0.89$ & $23.20 \pm 1.19$ & $0.735^{a}$ & $23.20 \pm 0.94$ & $23.22 \pm 1.16$ & $0.249^{c}$ \\
\hline $\mathrm{ACD}(\mathrm{mm})$ & $2.23 \pm 0.47$ & $2.27 \pm 0.50$ & $0.492^{a}$ & $2.28 \pm 0.46$ & $2.08 \pm 0.41$ & $0.040^{c}$ \\
\hline $\operatorname{LV}(\mathrm{mm})$ & $0.74 \pm 0.31$ & $0.89 \pm 0.27$ & $0.038^{a}$ & $0.70 \pm 0.35$ & $0.94 \pm 0.21$ & $0.010^{c}$ \\
\hline $\mathrm{AV}(\mathrm{mm})$ & $2.96 \pm 0.27$ & $3.17 \pm 0.47$ & $0.041^{a}$ & $2.99 \pm 0.26$ & $3.02 \pm 0.45$ & $0.418^{c}$ \\
\hline
\end{tabular}

ACG angle closure glaucoma, OAG open angle glaucoma, BCVA best-corrected visual acuity, LogMAR logarithm of the minimum angle of resolution, IOP, intraocular pressure, $A L$ axial length, $A C D$ anterior chamber depth, $L V$ lens vault, $A V$ anterior vault ${ }^{a}$ Independent t-test for stable subgroup and unstable subgroup

${ }^{b}$ Chi-square test for stable subgroup and unstable subgroup

${ }^{\mathrm{C}}$ Mann-Whitney $\mathrm{U}$ test for stable subgroup and unstable subgroup

IOP of combined group was $29.60 \pm 10.04 \mathrm{mmHg}$. It was significantly reduced to average of $13.35 \pm 3.12 \mathrm{mmHg}$ at 12 months postoperatively, and the postoperative need of glaucoma medications was significantly decreased. As in the previous studies, phacotrabeculectomy was found to be an effective treatment for lowering IOP.

In this study, we initially aimed to investigate prediction error after phacotrabeculectomy at 12 months postoperatively, the medical records of 120 patients in combined group and phaco-only group were analyzed. Among them, there were many patients who had medical records up to 2 years after the surgery, the data of 94 patients were additionally analyzed. And we found that there was no significant difference in prediction error between phacotrabeculectomy and phacoemulsification alone until 24 months after surgery. It means that phacotrabeculectomy was effective not only for IOP control but also for stable prediction error.

Previous studies reported that myopic shift occurs after phacotrabeculectomy compared to phacoemulsification alone [11, 23-25]. A decreased ACD after trabeculectomy causes a myopic shift, and a decreased AL after trabeculectomy causes a hyperopic shift, conversely. Some authors of these studies estimated that a decrease in ACD had a greater effect on refractive errors than a decrease in AL, leading to myopic shift. In the current study, since the prediction errors were compared using the absolute value of the difference of refractive errors,

Table 5 Factors associated with unstable prediction error in combined group at 12 months postoperatively

\begin{tabular}{|c|c|c|c|c|c|c|}
\hline \multirow[t]{2}{*}{ Variables } & \multicolumn{3}{|c|}{ Univariable analysis } & \multicolumn{3}{|c|}{ Multivariable analysis * } \\
\hline & $\overline{\mathrm{OR}}$ & $95 \% \mathrm{Cl}$ & $P$ value ${ }^{a}$ & $\overline{\mathrm{OR}}$ & $95 \% \mathrm{Cl}$ & $P$ value $^{a}$ \\
\hline Age, per 1 year older & 1.060 & $1.005-1.118$ & 0.031 & 1.069 & $1.007-1.135$ & 0.030 \\
\hline Male gender & 1.000 & $0.360-2.777$ & 1.000 & & & \\
\hline ACG & 1.000 & $0.360-2.777$ & 1.000 & & & \\
\hline Baseline BCVA, per 1 increase & 1.229 & $0.651-2.321$ & 0.524 & & & \\
\hline Baseline IOP, per $1 \mathrm{mmHg}$ increase & 0.953 & $0.903-1.006$ & 0.084 & 0.963 & $0.905-1.024$ & 0.229 \\
\hline $\mathrm{AL}$, per $1 \mathrm{~mm}$ increase & 1.090 & $0.667-1.782$ & 0.730 & & & \\
\hline $\mathrm{ACD}$, per $1 \mathrm{~mm}$ increase & 1.449 & $0.511-4.103$ & 0.485 & & & \\
\hline $\mathrm{LV}$, per $1 \mathrm{~mm}$ increase & 6.838 & $1.043-44.809$ & 0.045 & 5.687 & $1.195-27.064$ & 0.029 \\
\hline $\mathrm{AV}$, per $1 \mathrm{~mm}$ increase & 4.873 & $1.210-19.625$ & 0.047 & 6.231 & $0.755-51.425$ & 0.089 \\
\hline
\end{tabular}

$O R$ odds ratio, $C I$ confidence interval, $A C G$ angle closure glaucoma, $B C V A$ best-corrected visual acuity, $I O P$ intraocular pressure, $A L$ axial length, $A C D$ anterio chamber depth, $L V$ lens vault, $A V$ anterior vault

aLogistic regression analysis

* Only variables with a $P$ value of less than .10 in the univariable analysis were included in the multivariable model 
Table 6 Factors associated with unstable prediction error in combined group at 24 months postoperatively

\begin{tabular}{|c|c|c|c|c|c|c|}
\hline \multirow[t]{2}{*}{ Variables } & \multicolumn{3}{|c|}{ Univariable analysis } & \multicolumn{3}{|c|}{ Multivariable analysis * } \\
\hline & $\overline{\mathrm{OR}}$ & $95 \% \mathrm{Cl}$ & $P$ value ${ }^{a}$ & $\overline{\mathrm{OR}}$ & $95 \% \mathrm{Cl}$ & $P$ value ${ }^{a}$ \\
\hline Age, per 1 year older & 1.034 & $0.980-1.092$ & 0.226 & & & \\
\hline Male gender & 1.031 & $0.317-3.352$ & 0.959 & & & \\
\hline ACG & 2.078 & $0.640-6.744$ & 0.223 & & & \\
\hline Baseline BCVA, per 1 increase & 0.442 & $0.176-1.110$ & 0.082 & 0.460 & $0.157-1.349$ & 0.157 \\
\hline Baseline IOP, per $1 \mathrm{mmHg}$ increase & 0.991 & $0.933-1.054$ & 0.781 & & & \\
\hline $\mathrm{AL}$, per $1 \mathrm{~mm}$ increase & 1.022 & $0.589-1.771$ & 0.939 & & & \\
\hline$A C D$, per $1 \mathrm{~mm}$ increase & 0.346 & $0.088-1.362$ & 0.129 & & & \\
\hline $\mathrm{LV}$, per $1 \mathrm{~mm}$ increase & 21.309 & $1.813-250.393$ & 0.015 & 19.647 & $1.469-262.802$ & 0.024 \\
\hline $\mathrm{AV}$, per $1 \mathrm{~mm}$ increase & 1.260 & $0.270-5.878$ & 0.769 & & & \\
\hline
\end{tabular}

$O R$ odds ratio, $C l$ confidence interval, $A C G$ angle closure glaucoma, $B C V A$ best-corrected visual acuity, IOP intraocular pressure, $A L$ axial length, $A C D$ anterior chamber depth, $L V$ lens vault, $A V$ anterior vault

a Logistic regression analysis

* Only variables with a $P$ value of less than .10 in the univariable analysis were included in the multivariable model

we could not analyze whether myopic shift or hyperopic shift occurred after phacotrabeculectomy.

Law et al. [24] reported that K-value was increased after phacotrabeculectomy. Changes of not only ACD and AL but also K-value affect prediction errors, and if K-value increases, hyperopic shift may occur. Since this study did not measure postoperative K-value, ACD, and $\mathrm{AL}$, it was not possible to analyze which factors had more significant effect on prediction errors. However, we hypothesized that there was no significant difference in prediction errors between combined group and phaco-only group because the changes of $\mathrm{ACD}, \mathrm{AL}$, and K-value after phacotrabeculectomy had a global effect.

Most of the studies that focused the prediction errors of phacotrabeculectomy analyzed only short-term outcomes less than 6 months [11,18, 22-25], and one study reported by Chung et al. [12] had a limitation that the follow-up period of the control group was average of 4.81 months. Therefore, the present study is clinically significant because we analyzed long-term outcomes of prediction error up to 24 months after phacotrabeculectomy.

Tzu et al. [39] reported that the risk factor for prediction errors in combined cataract and glaucoma surgery included old age. Though the follow-up time was less than 6 months, glaucoma drainage device surgery was included in the combined group, it remains a meaningful result. Old age has been associated with structural changes of scleral collagen fiber and changes of $\mathrm{ACD}$ $[40,41]$, therefore, some studies reported that age of patients may affect the prediction errors after cataract surgery $[42,43]$. Our study also supports this result, as old age appeared to be a risk factor up to 12 months after phacotrabeculectomy. However, old age did not appear
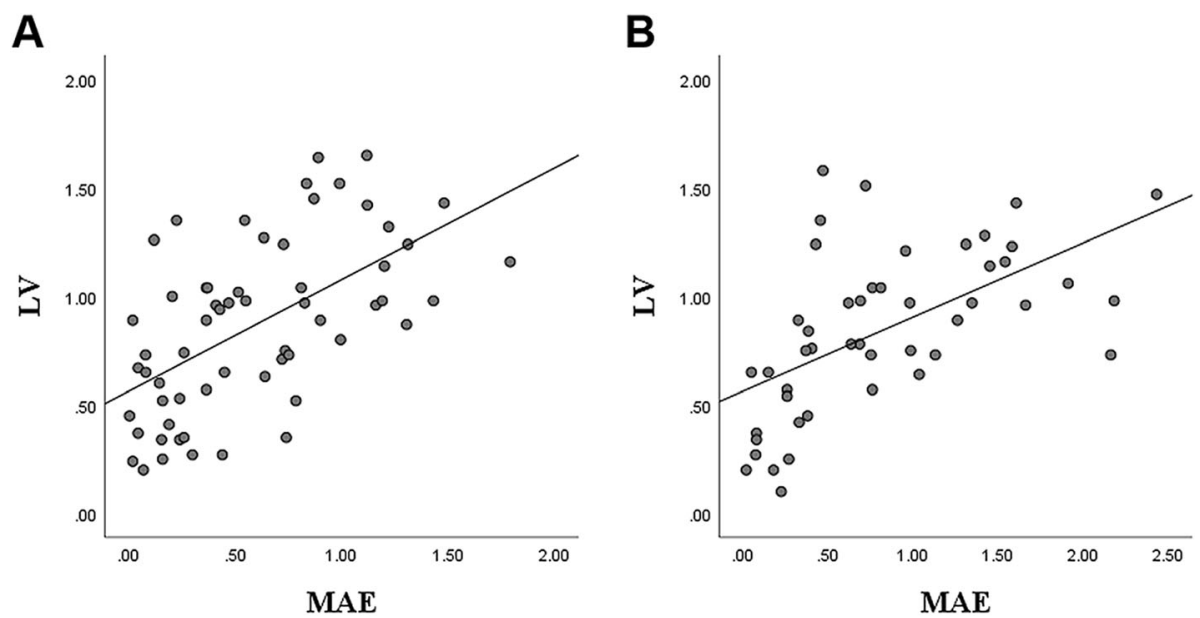

Fig. 1 Scatter plot of LV and MAE at 12 months postoperatively (a) and 24 months postoperatively (b). LV = lens vault; MAE = mean absolute error 

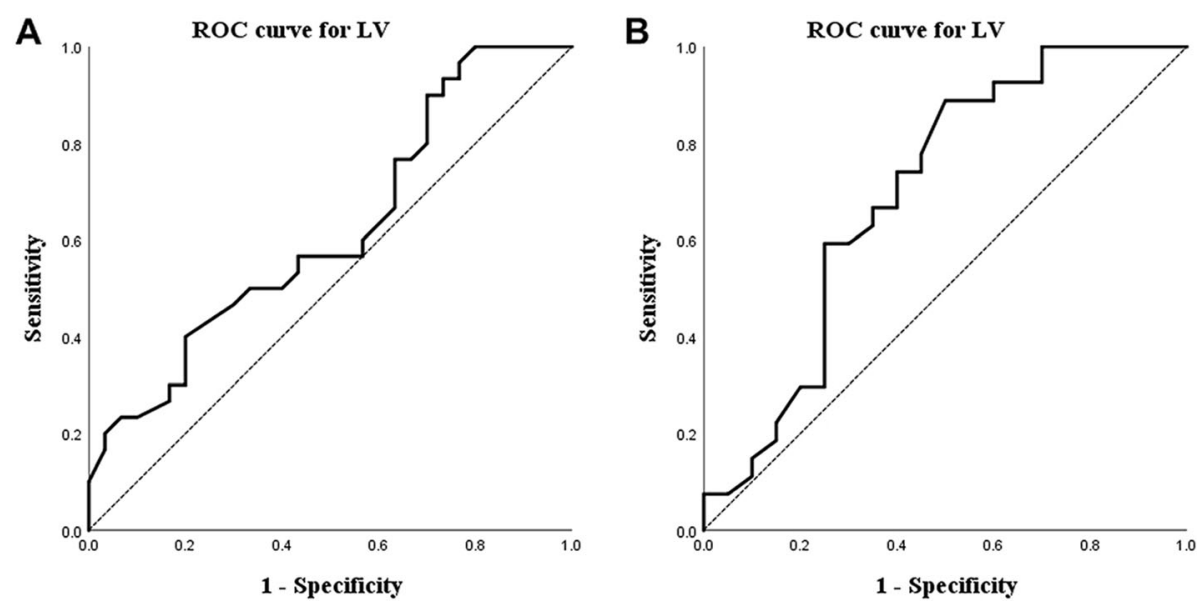

Fig. 2 Graphs showing the ROC curve of LV for unstable prediction error at 12 months postoperatively (a) and at 24 months postoperatively (b). The AUC of (a) was 0.619 and the AUC of (b) was 0.689. The cut-off value of LV was $0.855 \mathrm{~mm}$ in both (a) and (b). ROC = receiver operating characteristic; $L V=$ lens vault; $A \cup C=$ area under the curve

as a risk factor after 24 months of phacotrabeculectomy, which may be because of the relatively small number of patients.

Our group previously reported that one risk factor causing unstable prediction errors after cataract surgery in patients with glaucoma was large LV [44]. We speculated that large LV predispose to larger displacement of IOL position, resulting in unstable prediction error. In agreement with the previous report, in the current study, large LV was a risk factor that could cause unstable prediction errors up to 12 and 24 months after phacotrabeculectomy. Therefore, we suggest that LV plays an important role in predicting refractive errors after combined phacotrabeculectomy surgery in glaucoma patients as well as cataract surgery.

Ozaki et al. [45] reported that the LV of primary angle closure (PAC) patients was $1.034 \mathrm{~mm}$ on average, and that of normal people was $0.419 \mathrm{~mm}$, and $\mathrm{Hsia} \mathrm{YC}$ et al. [46] reported that LV of OAG patients was $0.55 \mathrm{~mm}$ on average. It has already been found that the increased LV is a risk factor of PAC and a predictive factor for refractive errors after cataract surgery in patients with glaucoma [44, 45], but there have been no studies analyzing the cut-off value of LV that can cause unstable prediction errors. We analyzed the long-term data after phacotrabeculectomy and found that a LV thickness of 0.855 or more was a risk factor for unstable prediction errors. Therefore, the surgeon should be very careful when operating patients with LV greater than $0.855 \mathrm{~mm}$ because unstable prediction errors can be obtained.

The current study has several limitations. First, we could not analyze which ocular parameters had a significant effect on refractive outcomes because some ocular parameters were not measured after surgery. Second, both patients with ACG and patients with OAG were included in the combined group, so the effect of angle status was not considered. Third, we did not consider digital massage or releasable suture removal that could affect refractive errors after phacotrabeculectomy. Forth, SRK-T formula was used instead of using the widely used Barrett or Haigis formulas, which is known to have high accuracy recently. Finally, we did not analyze the complications that could occur after phacotrabeculectomy because only patients without complication were included. In the future, larger and more long-term studies that consider these factors will be needed.

\section{Conclusions}

In conclusion, phacotrabeculectomy is an effective treatment that significantly reduces IOP and decreases the use of glaucoma medications in patients with cataract and medically uncontrolled glaucoma. There was no significant difference in prediction errors compared to phacoemulsification alone, and patients who underwent phacotrabeculectomy could also get stable prediction errors. However, the operator should be careful phacotrabeculectomy may increase in elderly patients or patients with large LV.

\section{Abbreviations}

AL: Axial length; ACD: Anterior chamber depth; IOL: Intraocular lens; IOP: Intraocular pressure; BCVA: Best-corrected visual acuity; ARK: Automated keratorefractometer; LogMAR: Logarithm of the minimum angle of resolution; SE: Spherical equivalent; AS-OCT: Anterior segment optical coherence tomography; LV: Lens vault; AV: Anterior vault; MAE: Mean absolute error; ROC: Receiver operating characteristic; AUC: Area under the curve; ACG: Angle closure glaucoma; OAG: Open angle glaucoma;

PAC: Primary angle closure

\section{Acknowledgements}

This research was supported by a grant of Patient-Centered Clinical Research Coordinating Center funded by the Ministry of Health \& Welfare, Republic of Korea (H119C0481, HC19C0276). The funding organization had no role in the design or conduct of this research. 


\section{Authors' contributions}

YSK, MSS, and SWP designed the study. YSK, HH, and YSJ collected the data and involved in the analysis. YSK wrote the first draft of the manuscript. YSK, MSS, and SWP reviewed and revised the manuscript and produced the final version. All authors have read and approved the final manuscript.

\section{Funding}

No funding was received by any of the authors in the writing of this manuscript.

\section{Availability of data and materials}

The datasets generated and/or analyzed during the current study are not publicly available to protect patient identify and confidentiality but are available from the corresponding author on reasonable request.

\section{Ethics approval and consent to participate}

This study adhered to the Declaration of Helsinki and was approved by the Chonnam National University Hospital Institutional Review Board. Written informed consents were obtained after the subjects were fully informed of the purposes of this study.

\section{Consent for publication}

Not applicable (no identifying patient data).

\section{Competing interests}

The authors declare that they have no competing interests.

\section{Received: 12 July 2020 Accepted: 20 January 2021}

Published online: 26 January 202

\section{References}

1. Husain R, Li W, Gazzard G, Foster PJ, Chew PT, Oen FT, et al. Longitudinal changes in anterior chamber depth and axial length in Asian subjects after trabeculectomy surgery. Br J Ophthalmol. 2013;97:852-6.

2. Cashwell LF, Martin CA. Axial length decrease accompanying successful glaucoma filtration surgery. Ophthalmology. 1999;106:2307-11.

3. Kook MS, Kim HB, Lee SU. Short-term effect of mitomycin-C augmented trabeculectomy on axial length and corneal astigmatism. J Cataract Refract Surg. 2001;27:518-23.

4. Hugkulstone CE. Changes in keratometry following trabeculectomy. $\mathrm{Br}$ J Ophthalmol. 1991;75:217-8

5. Rosen WJ, Mannis MJ, Brandt JD. The effect of trabeculectomy on corneal topography. Ophthalmic Surg. 1992;23:395-8.

6. Claridge KG, Galbraith JK, Karmel V, Bates AK. The effect of trabeculectomy on refraction, keratometry and corneal topography. Eye (Lond). 1995;9(Pt 3): 292-8.

7. Olsen T. Sources of error in intraocular lens power calculation. J Cataract Refract Surg. 1992;18:125-9.

8. Hoffer KJ. The Hoffer Q formula: a comparison of theoretic and regression formulas. J Cataract Refract Surg. 1993;19:700-12.

9. Olsen T, Corydon L, Gimbel H. Intraocular lens power calculation with an improved anterior chamber depth prediction algorithm. J Cataract Refract Surg. 1995;21:313-9.

10. Norrby NE, Koranyi G. Prediction of intraocular lens power using the lens haptic plane concept. J Cataract Refract Surg. 1997;23:254-9.

11. Chan JCH, Lai JSM, Tham CCY. Comparison of postoperative refractive outcome in phacotrabeculectomy and phacoemulsification with posterior chamber intraocular lens implantation. J Glaucoma. 2006;15:26-9.

12. Chung JK, Wi JM, Lee KB, Ahn BH, Hwang YH, Kim M, et al. Long-term comparison of postoperative refractive outcomes between phacotrabeculectomy and phacoemulsification. J Cataract Refract Surg. 2018:44:964-70.

13. Choi YJ, Rhee DJ, Choi K-R. Treatment outcome of Phacotrabeculectomy and trabeculectomy in patients with cataracts and Glaucoma. J Korean Ophthalmol Soc. 2011;52:1308.

14. Hong S, Park K, Ha SJ, Yeom HY, Seong GJ, Hong YJ. Long-term intraocular pressure control of trabeculectomy and triple procedure in primary open angle Glaucoma and chronic primary angle closure Glaucoma. Ophthalmologica. 2007;221:395-401.
15. Chen DZ, Koh V, Sng C, Aquino MC, Chew P. Complications and outcomes of primary Phacotrabeculectomy with Mitomycin C in a multi-ethnic Asian population. PLoS One. 2015;10:e0118852.

16. Li S-W, Chen Y, Wu Q, Lu B, Wang W-Q, Fang J. Angle parameter changes of phacoemulsification and combined phacotrabeculectomy for acute primary angle closure. Int J Ophthalmol. 2015:8:742-7.

17. El Sayed YM, Elhusseiny AM, Albalkini AS, El Sheikh RH, Osman MA. Mitomycin C-augmented Phacotrabeculectomy versus phacoemulsification in primary angle-closure Glaucoma: a randomized controlled study. J Glaucoma. 2019;28:911-5.

18. Vaideanu D, Mandal K, Hildreth A, Fraser SG, Phelan PS. Visual and refractive outcome of one-site phacotrabeculectomy compared with temporal approach phacoemulsification. Clin Ophthalmol. 2008;2:569-74.

19. Murchison JF, Shields MB. Limbal-based vs fornix-based conjunctival flaps in combined extracapsular cataract surgery and glaucoma filtering procedure. Am J Ophthalmol. 1990;109:709-15.

20. Krupin T, Feitl ME, Bishop Kl. Postoperative intraocular pressure rise in openangle glaucoma patients after cataract or combined cataract-filtration surgery. Ophthalmology. 1989;96:579-84.

21. Shin DH, Iskander NG, Ahee JA, Singal IP, Kim C, Hughes BA, et al. Longterm filtration and visual field outcomes after primary glaucoma triple procedure with and without mitomycin-C. Ophthalmology. 2002;109:160711.

22. Lee DE, Kim JL. Comparison of postoperative refractive outcomes after Phacotrabeculectomy and phacoemulsification subsequent to trabeculectomy. J Korean Ophthalmol Soc. 2017;58:1349.

23. Bae HW, Lee YH, Kim DW, Lee T, Hong S, Seong GJ, et al. Effect of trabeculectomy on the accuracy of intraocular lens calculations in patients with open-angle glaucoma. Clin Exp Ophthalmol. 2016:44:465-71.

24. Law SK, Mansury AM, Vasudev D, Caprioli J. Effects of combined cataract surgery and trabeculectomy with mitomycin C on ocular dimensions. $\mathrm{Br}$ J Ophthalmol. 2005:89:1021-5.

25. Ong C, Nongpiur M, Peter $L$, Perera SA. Combined approach to phacoemulsification and trabeculectomy results in less ideal refractive outcomes compared with the sequential approach. J Glaucoma. 2016;25: e873-8.

26. Kim YK, Yoo BW, Kim HC, Aung T, Park KH. Relative lens vault in subjects with angle closure. BMC Ophthalmol. 2014;14:93.

27. Vizzeri G, Weinreb RN. Cataract surgery and glaucoma. Curr Opin Ophthalmol. 2010:21:20-4

28. Young CEC, Seibold LK, Kahook MY. Cataract surgery and intraocular pressure in glaucoma. Curr Opin Ophthalmol. 2020;31:15-22.

29. Kung JS, Choi DY, Cheema AS, Singh K. Cataract surgery in the glaucoma patient. Middle East Afr J Ophthalmol. 2015;22:10-7.

30. Lam DSC, Leung DYL, Tham CCY, Li FCH, Kwong YYY, Chiu TYH, et al. Randomized trial of early phacoemulsification versus peripheral iridotomy to prevent intraocular pressure rise after acute primary angle closure. Ophthalmology. 2008;115:1134-40.

31. Jimenez-Roman J, Lazcano-Gomez G, Martínez-Baez K, Turati M, GulíasCañizo R, Hernández-Zimbrón LF, et al. Effect of phacoemulsification on intraocular pressure in patients with primary open angle glaucoma and pseudoexfoliation glaucoma. Int J Ophthalmol. 2017;10:1374-8.

32. Chen PP, Lin SC, Junk AK, Radhakrishnan S, Singh K, Chen TC. The effect of phacoemulsification on intraocular pressure in Glaucoma patients: a report by the American Academy of ophthalmology. Ophthalmology. 2015;122: 1294-307.

33. Shingleton BJ, Pasternack JJ, Hung JW, O'Donoghue MW. Three and five year changes in intraocular pressures after clear corneal phacoemulsification in open angle glaucoma patients, glaucoma suspects, and normal patients. I Glaucoma. 2006:15:494-8.

34. Sihota R, Lakshmaiah NC, Agarwal HC, Pandey RM, Titiyal JS. Ocular parameters in the subgroups of angle closure glaucoma. Clin Exp Ophthalmol. 2000;28:253-8.

35. Kugelberg M, Lundström M. Factors related to the degree of success in achieving target refraction in cataract surgery: Swedish National Cataract Register study. J Cataract Refract Surg. 2008:34:1935-9.

36. Wang F, Zhi-Hong W. Phacoemulsification versus combined phacotrabeculectomy in the treatment of primary angle-closure glaucoma with cataract: a meta-analysis. Int J Ophthalmol. 2016;9:597-603.

37. Tham CCY, Kwong YYY, Leung DYL, Lam SW, Li FCH, Chiu TYH, et al. Phacoemulsification versus combined phacotrabeculectomy in medically 
controlled chronic angle closure glaucoma with cataract. Ophthalmology. 2008;115:2167-73.

38. Tham CCY, Kwong YYY, Leung DYL, Lam SW, Li FCH, Chiu TYH, et al. Phacoemulsification versus combined phacotrabeculectomy in medically uncontrolled chronic angle closure glaucoma with cataracts. Ophthalmology. 2009;116:725-31.

39. Tzu JH, Shah CT, Galor A, Junk AK, Sastry A, Wellik SR. Refractive outcomes of combined cataract and glaucoma surgery. J Glaucoma. 2015;24:161-4.

40. Coudrillier B, Pijanka J, Jefferys J, Sorensen T, Quigley HA, Boote C, et al. Effects of age and diabetes on scleral stiffness. J Biomech Eng 2015;137:0710071-07100710

41. Hashemi H, Yekta A, Khodamoradi F, Aghamirsalim M, Asharlous A, Assadpour $\mathrm{M}$, et al. Anterior chamber indices in a population-based study using the Pentacam. Int Ophthalmol. 2019:39:2033-40.

42. Nuzzi G, Cantù C, De Giovanni MA. Older age as risk factor for deviation from emmetropia in pseudophakia. Eur J Ophthalmol. 2001;11: 133-8.

43. Hayashi K, Ogawa S, Yoshida M, Yoshimura K. Influence of patient age on intraocular Lens power prediction error. Am J Ophthalmol. 2016;170: 232-7.

44. Kim YC, Sung MS, Heo H, Park SW. Anterior segment configuration as a predictive factor for refractive outcome after cataract surgery in patients with glaucoma. BMC Ophthalmol. 2016;16:179.

45. Ozaki M, Nongpiur ME, Aung T, He M, Mizoguchi T. Increased lens vault as a risk factor for angle closure: confirmation in a Japanese population. Graefes Arch Clin Exp Ophthalmol. 2012;250:1863-8.

46. Hsia YC, Moghimi S, Coh P, Chen R, Masis M, Lin SC. Anterior segment parameters as predictors of intraocular pressure reduction after phacoemulsification in eyes with open-angle glaucoma. J Cataract Refract Surg. 2017;43:879-85.

\section{Publisher's Note}

Springer Nature remains neutral with regard to jurisdictional claims in published maps and institutional affiliations.

Ready to submit your research? Choose BMC and benefit from:

- fast, convenient online submission

- thorough peer review by experienced researchers in your field

- rapid publication on acceptance

- support for research data, including large and complex data types

- gold Open Access which fosters wider collaboration and increased citations

- maximum visibility for your research: over $100 \mathrm{M}$ website views per year

At BMC, research is always in progress.

Learn more biomedcentral.com/submissions 